
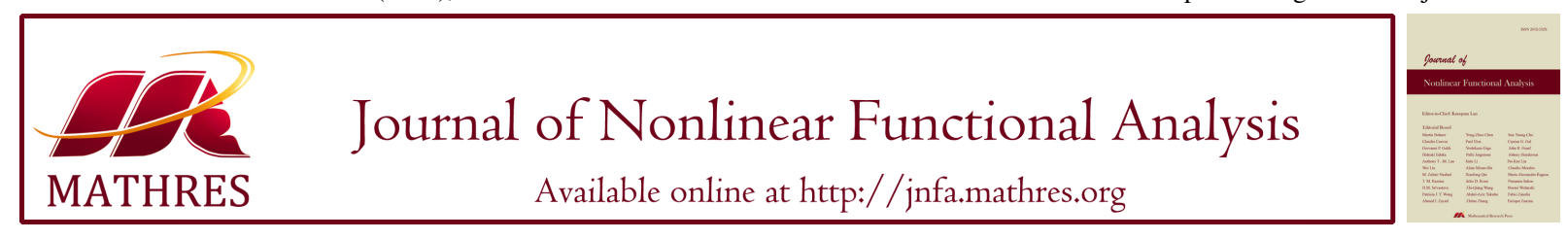

\title{
A UNIFIED APPROACH TO FULLY THIRD ORDER NONLINEAR BOUNDARY VALUE PROBLEMS
}

\author{
QUANG A. DANG ${ }^{1, *}$, QUANG LONG DANG ${ }^{2}$ \\ ${ }^{1}$ Centre for Informatics and Computing, VAST, 18 Hoang Quoc Viet, Cau Giay, Hanoi, Vietnam \\ ${ }^{2}$ Institute of Information Technology, VAST, 18 Hoang Quoc Viet, Cau Giay, Hanoi, Vietnam
}

\begin{abstract}
In this paper, we propose a unified approach to investigate boundary value problems for fully third order differential equations. By this approach, we establish the existence, uniqueness, positivity and monotonicity of solutions and the convergence of an iterative method for approximating the solutions under some easily verified conditions in bounded domains. Examples are presented to illustrate our results.
\end{abstract}

Keywords. Fully third order nonlinear equation; Existence and uniqueness; Positivity and monotonicity; Iterative method.

\section{INTRODUCTION}

In recent years, the boundary value problems (BVPs) for the third order nonlinear differential equation

$$
u^{\prime \prime \prime}(t)=f\left(t, u(t), u^{\prime}(t), u^{\prime \prime}(t)\right), \quad 0<t<1
$$

have attracted attention from many researchers. A number of results are devoted to the existence, uniqueness and positivity of solutions of the problems with different boundary conditions. The methods for investigating qualitative aspects of the problems are diverse, including the method of lower and upper solutions and monotone technique $[1,2,3,4,5,6]$, the Leray-Schauder continuation principle [7], the fixed point theory on cones [8] and so on. It should be emphasized that in the above works there is an essential assumption that the function $f(t, x, y, z):[0,1] \times \mathbb{R}^{3} \rightarrow \mathbb{R}$ satisfies a Nagumo-type condition on the last two variables [9], or linear growth in $x, y, z$ at infinity [7], or some complicated conditions including monotone increase in each of $x$ and $y$ [10], or one-sided Lipschitz condition in $x$ for $f=f(t, x)$ [6] and in $x, y$ for $f=f(t, x, y)$ [4]. Sun, Zhao and Li [11] studied the existence of monotone positive solutions of the BVP for the case $f=f(u(t))$ under conditions, which are difficult to be verified.

\footnotetext{
${ }^{*}$ Corresponding author.
}

E-mail addresses: dangquanga @ cic.vast.vn (Q.A. Dang), dqlong88@gmail.com (Q.L. Dang).

Received August 26, 2019; Accepted February 22, 2020. 
In this paper, motivated greatly by the above-mentioned results, we propose a unified efficient method to investigate the solvability and approximation of BVPs for the fully third order equation (1.1) with general boundary conditions

$$
\begin{aligned}
& B_{1}[u]=\alpha_{1} u(0)+\beta_{1} u^{\prime}(0)+\gamma_{1} u^{\prime \prime}(0)=0, \\
& B_{2}[u]=\alpha_{2} u(0)+\beta_{2} u^{\prime}(0)+\gamma_{2} u^{\prime \prime}(0)=0, \\
& B_{3}[u]=\alpha_{3} u(1)+\beta_{3} u^{\prime}(1)+\gamma_{3} u^{\prime \prime}(1)=0,
\end{aligned}
$$

and

$$
\begin{aligned}
& B_{1}[u]=\alpha_{1} u(0)+\beta_{1} u^{\prime}(0)+\gamma_{1} u^{\prime \prime}(0)=0, \\
& B_{2}[u]=\alpha_{2} u(1)+\beta_{2} u^{\prime}(1)+\gamma_{2} u^{\prime \prime}(1)=0, \\
& B_{3}[u]=\alpha_{3} u(1)+\beta_{3} u^{\prime}(1)+\gamma_{3} u^{\prime \prime}(1)=0,
\end{aligned}
$$

such that

$$
\operatorname{Rank}\left(\begin{array}{cccccc}
\alpha_{1} & \beta_{1} & \gamma_{1} & 0 & 0 & 0 \\
\alpha_{2} & \beta_{2} & \gamma_{2} & 0 & 0 & 0 \\
0 & 0 & 0 & \alpha_{3} & \beta_{3} & \gamma_{3}
\end{array}\right)=3
$$

The boundary conditions (1.2) include as particular cases the boundary conditions considered in $[3,4,6,7,10]$, meanwhile the boundary conditions (1.3) include as particular cases the boundary conditions considered in $[3,9]$. Notice that the boundary conditions of the form (1.3) can be transformed to the boundary conditions of the form (1.2) by changing variable $t=1-s$.

To investigate the BVP (1.1)-(1.2) as the BVP (1.1), (1.3), we use a new approach based on the reduction of them to operator equations for the nonlinear terms but not for the functions to be sought. This approach was used by ourselves to some boundary value problems for fourth and sixth order nonlinear equations in very recent works $[12,13,14,15,16,17]$ and for nonlinear biharmonic equation [18]. Besides, the approach was also used in [19, 20]. By this approach, we establish the existence, uniqueness, positivity and monotonicity of solutions and the convergence of the iterative method for approximating the solutions of the problems (1.1)-(1.2) under some easily verified conditions in bounded domains. Examples are presented to illustrate our results.

\section{EXISTENCE RESULTS}

Since problem (1.1)-(1.2) and problem (1.1), (1.3) are completely similar, we only consider the first one below. For the sake of convenience, we rewrite problem (1.1)-(1.2) in the form

$$
\begin{aligned}
u^{\prime \prime \prime}(t) & =f\left(t, u(t), u^{\prime}(t), u^{\prime \prime}(t)\right), \quad 0<t<1, \\
B_{1}[u] & =B_{2}[u]=B_{3}[u]=0,
\end{aligned}
$$

where $B_{1}[u], B_{2}[u], B_{3}[u]$ are defined by (1.2). We associate this problem with an operator equation as follows. For functions $\varphi(x) \in C[0,1]$, we consider the nonlinear operator $A$ defined by

$$
(A \varphi)(t)=f\left(t, u(t), u^{\prime}(t), u^{\prime \prime}(t)\right),
$$

where $u(t)$ is the solution of the problem

$$
\begin{aligned}
u^{\prime \prime \prime}(t) & =\varphi(t), \quad 0<t<1 \\
B_{1}[u] & =B_{2}[u]=B_{3}[u]=0
\end{aligned}
$$


provided that it is uniquely solvable. It is easy to verify the following.

Proposition 2.1. If the function $\varphi(t)$ is a fixed point of the operator $A$, i.e., $\varphi(t)$ is a solution of the operator equation

$$
A \varphi=\varphi,
$$

then the function $u(t)$ determined from boundary value problem (2.3) solves problem (2.1). Conversely, if $u(t)$ is a solution of boundary value problem (2.1), then

$$
\varphi(t)=f\left(t, u(t), u^{\prime}(t), u^{\prime \prime}(t)\right)
$$

is a fixed point of the operator A defined above by (2.2)-(2.3).

Thus, the solution of the original problem (2.1) is reduced to the solution of operator equation (2.4). Now, we consider problem (2.3). Suppose that the Green function of it exists and is denoted by $G(t, s)$. Then the unique solution of the problem is represented in the form

$$
u(t)=\int_{0}^{1} G(t, s) \varphi(s) d s .
$$

By differentiation of both sides of the above formula, we obtain

$$
u^{\prime}(t)=\int_{0}^{1} G_{1}(t, s) \varphi(s) d s, \quad u^{\prime \prime}(t)=\int_{0}^{1} G_{2}(t, s) \varphi(s) d s,
$$

where $G_{1}(t, s)=G_{t}^{\prime}(t, s)$ is a function continuous in the square $Q=[0,1]^{2}$ and $G_{2}(t, s)=G_{t t}^{\prime \prime}(t, s)$ is continuous in the square $Q$ except for the line $t=s$. Further, let

$$
\begin{aligned}
& \max _{0 \leq t \leq 1} \int_{0}^{1}|G(t, s)| d s=M_{0} \\
& \max _{0 \leq t \leq 1} \int_{0}^{1}\left|G_{1}(t, s)\right| d s=M_{1}, \max _{0 \leq t \leq 1} \int_{0}^{1}\left|G_{2}(t, s)\right| d s=M_{2} .
\end{aligned}
$$

Next, for each fixed real number $M>0$, we introduce the domain

$$
\mathscr{D}_{M}=\left\{(t, x, y, z)|0 \leq t \leq 1,| x\left|\leq M_{0} M,\right| y\left|\leq M_{1} M,\right| z \mid \leq M_{2} M\right\} .
$$

As usual, we denote by $B[O, M]$ the closed ball of radius $M$ centered at 0 in the space of continuous in $[0,1]$ functions, namely,

$$
B[O, M]=\{\varphi \in C[0,1] \mid\|\varphi\| \leq M\},
$$

where

$$
\|\varphi\|=\max _{0 \leq t \leq 1}|\varphi(t)| .
$$

Theorem 2.2 (Existence of solutions). Suppose that there exists a number $M>0$ such that the function $f(t, x, y, z)$ is continuous and bounded by $M$ in the domain $\mathscr{D}_{M}$, i.e.,

$$
|f(t, x, y, z)| \leq M
$$

for any $(t, x, y, z) \in \mathscr{D}_{M}$.

Then, problem (2.1) has a solution $u(t)$ satisfying

$$
|u(t)| \leq M_{0} M,\left|u^{\prime}(t)\right| \leq M_{1} M,\left|u^{\prime \prime}(t)\right| \leq M_{2} M \text { for any } 0 \leq t \leq 1 .
$$


Proof. Having in mind Proposition 2.1 the theorem can be proved if we show that the operator $A$ associated with the problem (2.1) has a fixed point. For this purpose, it is not difficult to show that $A$ maps the closed ball $B[0, M]$ into itself. Next, from the compactness of integral operators (2.5), (2.6), which put each $\varphi \in C[0,1]$ in correspondence to the functions $u, u^{\prime}, u^{\prime \prime}$, respectively [21, Sec. 31] (see APPENDIX) and the continuity of the function $f(t, x, y, z)$, it follows that $A$ is a compact operator in the Banach space $C[0,1]$. By the Schauder Fixed Point Theorem [22], the operator $A$ has a fixed point in $B[0, M]$. The estimates that (2.9) holds due to the equalities (2.5), (2.6) and (2.7). The theorem is proved.

Now suppose that the Green function $G(x, t)$ and its first derivative $G_{1}(x, t)$ are of constant signs in the square $Q=[0,1]^{2}$. Let us adopt the following convention for simplification of writing. For a function $H(x, t)$ with a constant sign in the square $Q$, we define

$$
\sigma(H)=\operatorname{sign}(H(t, s))= \begin{cases}1, & \text { if } H(t, s) \geq 0, \\ -1, & \text { if } H(t, s)<0 .\end{cases}
$$

In order to investigate the existence of positive solutions of problem (1.1)-(1.2), we introduce the notations

$$
\begin{aligned}
\mathscr{D}_{M}^{+}=\{(t, x, y, z) \mid & 0 \leq t \leq 1,0 \leq x \leq M_{0} M, \\
0 & \left.\leq \sigma(G) \sigma\left(G_{1}\right) y \leq M_{1} M,|z| \leq M_{2} M\right\}
\end{aligned}
$$

and

$$
S_{M}=\{\varphi \in C[0,1] \mid 0 \leq \sigma(G) \varphi \leq M\} .
$$

Theorem 2.3 (Existence of positive solution). Suppose that there exists a number $M>0$ such that the function $f(t, x, y, z)$ is continuous and

$$
0 \leq \sigma(G) f(t, x, y, z) \leq M
$$

for any $(t, x, y, z) \in \mathscr{D}_{M}^{+}$. Then, problem (1.1)-(1.2) has a monotone nonnegative solution $u(t)$ satisfying

$$
0 \leq u(t) \leq M_{0} M, 0 \leq \sigma(G) \sigma\left(G_{1}\right) u^{\prime}(t) \leq M_{1} M,\left|u^{\prime \prime}(t)\right| \leq M_{2} M .
$$

In addition, if $\sigma(G) \sigma\left(G_{1}\right)=1$, then the problem has a nonnegative, increasing solution, and if $\sigma(G) \sigma\left(G_{1}\right)=-1$, then the problem has a nonnegative, decreasing solution. Besides, if $f(t, 0,0,0) \not \equiv 0$ for $t \in(0,1)$, then the solution is positive.

Proof. The proof of the existence of monotone nonnegative solution of the problem is similar to that of the solution in Theorem 2.2 with the replacements of $\mathscr{D} \mathscr{M}$ by $\mathscr{D}_{M}^{+}, B[0, M]$ by $S_{M}$ and the condition (2.8) by the condition (2.10). From the estimates (2.11), it is obvious that if $\sigma(G) \sigma\left(G_{1}\right)=1$, then $u^{\prime}(t) \geq 0$. Consequently, the solution is increasing function, otherwise, if $\sigma(G) \sigma\left(G_{1}\right)=-1$, then $u^{\prime}(t) \leq 0$. Therefore, the solution is decreasing function. Moreover, if $f(t, 0,0,0) \not \equiv 0$ for $t \in(0,1)$, then $u=0$ cannot be the solution of the problem. Therefore, it must be positive. The theorem is proved.

Theorem 2.4 (Existence and uniqueness of solution). Assume that there exist numbers $M, L_{0}, L_{1}$, $L_{2} \geq 0$ such that

$$
\begin{gathered}
|f(t, x, y, z)| \leq M \\
\left|f\left(t, x_{2}, y_{2}, z_{2}\right)-f\left(t, x_{1}, y_{1}, z_{1}\right)\right| \leq L_{0}\left|x_{2}-x_{1}\right|+L_{1}\left|y_{2}-y_{1}\right|+L_{2}\left|z_{2}-z_{1}\right|
\end{gathered}
$$


for any $(t, x, y, z),\left(t, x_{i}, y_{i}, z_{i}\right) \in \mathscr{D}_{M}(i=1,2)$ and

$$
q:=L_{0} M_{0}+L_{1} M_{1}+L_{2} M_{2}<1 .
$$

Then, problem (1.1)-(1.2) has a unique solution $u(t)$ such that $|u(t)| \leq M_{0} M,\left|u^{\prime}(t)\right| \leq M_{1} M$, $\left|u^{\prime \prime}(t)\right| \leq M_{2} M$ for any $0 \leq t \leq 1$.

Proof. It is easy to show that under the conditions of the theorem, the operator $A$ associated with the problem (1.1),(1.2) is a contraction mapping from the closed ball $B[0, M]$ into itself. By the contraction principle the operator $A$ has a unique fixed point in $B[O, M]$, which corresponds to a unique solution $u(t)$ of the problem (1.1),(1.2).

The estimates for $u(t)$ and its derivatives are obtained as in Theorem 2.2. Thus, the theorem is proved.

Analogously, we have the following theorem for the existence and uniqueness of positive solutions of problem (1.1)-(1.2).

Theorem 2.5 (Existence and uniqueness of positive solution). Assume that all the conditions of Theorem 2.3 are satisfied in the domain $\mathscr{D}_{M}^{+}$. Moreover, assume that there exist numbers $L_{0}, L_{1}, L_{2} \geq 0$ such that the function $f(t, x, y, z)$ satisfies the Lipschitz conditions (2.12), (2.13). Then, problem (1.1)-(1.2) has a unique monotone nonnegative solution $u(t)$ satisfying (2.11). Besides, if $f(t, 0,0,0) \not \equiv 0$ for $t \in(0,1)$, then the solution is positive.

Remark 2.6. Due to the representation (2.6) for $u^{\prime \prime}(t)$, based on the sign of $G(t, s)$ and $G_{2}(t, s)$, we can conclude the convexity or concavity of the solutions of problem (2.1).

\section{THE ITERATIVE METHOD}

Consider the following iterative method for solving problem (1.1)-(1.2):

(1) Given a starting approximation $\varphi_{0} \in B[0, M]$, say

$$
\varphi_{0}(t)=0,
$$

(2) with $\varphi_{k}(k=0,1, \ldots)$, compute

$$
\begin{gathered}
u_{k}(t)=\int_{0}^{1} G(t, s) \varphi_{k}(s) d s, \\
y_{k}(t)=u_{k}^{\prime}(t), z_{k}(t)=u_{k}^{\prime \prime}(t),
\end{gathered}
$$

or equivalently,

$$
\begin{aligned}
& y_{k}(t)=\int_{0}^{1} G_{1}(t, s) \varphi_{k}(s) d s, \\
& z_{k}(t)=\int_{0}^{1} G_{2}(t, s) \varphi_{k}(s) d s,
\end{aligned}
$$

(3) update the new approximation

$$
\varphi_{k+1}(t)=f\left(t, u_{k}(t), y_{k}(t), z_{k}(t)\right),
$$

and set

$$
p_{k}=\frac{q^{k}}{1-q}\left\|\varphi_{1}-\varphi_{0}\right\|
$$


Theorem 3.1 (Convergence). Under the assumptions of Theorem 2.4, the above iterative method converges and there hold the estimates

$$
\left\|u_{k}-u\right\| \leq M_{0} p_{k}, \quad\left\|u_{k}^{\prime}-u^{\prime}\right\| \leq M_{1} p_{k}, \quad\left\|u_{k}^{\prime \prime}-u^{\prime \prime}\right\| \leq M_{2} p_{k},
$$

where $u$ is the exact solution of problem (1.1)-(1.2), and $M_{0}, M_{1}, M_{2}$ are given by (2.7).

Proof. Indeed, the above iterative process is the successive approximation of the fixed point of the operator $A$ associated with problem (1.1)-(1.2). Therefore, it converges with the rate of geometric progression and there is the estimate

$$
\left\|\varphi_{k}-\varphi\right\| \leq p_{k}
$$

where $\varphi$ is the fixed point of $A$. Taking into account the representations (2.5), (2.6), (3.2), (3.4) and the formulas (2.7), from the above estimate, we obtain the estimates (3.6). Thus, the theorem is proved.

In many problems, when the Green function and its derivatives have constant sign, and the right-hand side function $f(t, x, y, z)$ is monotone in variables $x, y, z$, we can establish the monotonicity of the sequence of approximations $u_{k}(t)$. Below, we consider a particular case, which will be met in some examples in the next section.

Theorem 3.2 (Monotonicity). Consider problem (1.1)-(1.2), where the Green function $G(t, s)$ and its derivative $G_{1}(t, s)$ are nonpositive in the square $Q=[0,1]^{2}$, the function $f=f(t, x, y) \leq 0$ is decreasing in $x, y$ for $x, y \geq 0$. Then the sequence of approximations $u_{k}(t)$ generated by the above iterative process is increasing, i.e.

$$
0=u_{0}(t) \leq u_{1}(t) \leq \ldots \leq u_{k}(t) \leq \ldots, \quad t \in[0,1] .
$$

Proof. Indeed, starting from $\varphi_{0}=0$, we obtain from the iterative process (3.1)-(3.5) that $u_{0}=$ $0, y_{0}=0$. Since $f=f(t, x, y) \leq 0$, we have $\varphi_{1}=f(t, 0,0) \leq 0$. Therefore, $u_{1}(t)=\int_{0}^{1} G(t, s) \varphi_{1}(s)$ $d s \geq 0$ due to $G(t, s) \leq 0$. Analogously, $y_{1}(t) \geq 0$. Thus, $u_{1} \geq u_{0}, y_{1} \geq y_{0}$. Due to the decrease of $f(t, x, y)$ in $x, y$, we have $\varphi_{2}(t)=f\left(t, u_{1}, y_{1}\right) \leq f\left(t, u_{0}, y_{0}\right)=\varphi_{1}(t)$. Therefore, from the formulas for computing $u_{2}(t), y_{2}(t)$, it follows that $u_{2} \geq u_{1}, y_{2} \geq y_{1}$. Repeating the above argument, we obtain (3.8). The theorem is proved.

\section{Some PARTICULAR CASES AND EXAMPLES}

Consider some particular cases of the general BVP (1.1)-(1.2) and BVP (1.1), (1.3), which cover the problems studied by other authors using different methods. For each case, the theoretical results obtained in the previous section will be illustrated on examples, including some examples considered before by other authors. In numerical realization of the proposed iterative method, for computing definite integrals the trapezium formula with second order accuracy is used. In all examples, numerical computations are performed on the uniform grid on the interval $[0,1]$ with the gridsize $h=0.01$ until $\left\|\varphi_{k}-\varphi_{k-1}\right\| \leq 10^{-6}$. The number of iterations for reaching the above accuracy will be indicated. From the particular cases together with examples, it will be clear of the efficiency of the proposed approach to BVPs for nonlinear third order differential equations by the reduction of them to operator equations for the nonlinear terms. 
4.1. Case 1. Consider the problem

$$
\begin{aligned}
u^{(3)}(t) & =f\left(t, u(t), u^{\prime}(t), u^{\prime \prime}(t)\right), \quad 0<t<1, \\
u(0) & =u^{\prime}(0)=u^{\prime}(1)=0 .
\end{aligned}
$$

For the case $f=f(t, u(t))$ in [6], using the lower and upper solutions method and the fixed point theorem on cones, Yao and Feng established several results of solutions and positive solutions. For the case $f=f\left(t, u(t), u^{\prime}(t)\right)$ in [4] Feng and Liu also obtained existence results with the aid of the upper and lower solutions method and a new maximum principle. It should be emphasized that the results of these two works are pure existence but not uniqueness.

The Green function associated with the considered problem has the form

$$
G(t, s)=\left\{\begin{array}{l}
\frac{s}{2}\left(t^{2}-2 t+s\right), \quad 0 \leq s \leq t \leq 1, \\
\frac{t^{2}}{2}(s-1), \quad 0 \leq t \leq s \leq 1
\end{array}\right.
$$

After differentiation of $G(t, s)$, we obtain

$$
\begin{aligned}
& G_{1}(t, s)= \begin{cases}s(t-1), & 0 \leq s \leq t \leq 1, \\
t(s-1), & 0 \leq t \leq s \leq 1,\end{cases} \\
& G_{2}(t, s)= \begin{cases}s, & 0 \leq s \leq t \leq 1, \\
s-1, & 0 \leq t \leq s \leq 1\end{cases}
\end{aligned}
$$

It is obvious that

$$
G(t, s) \leq 0, G_{1}(t, s) \leq 0,0 \leq t, s \leq 1
$$

and then

$$
\begin{aligned}
M_{0} & =\max _{0 \leq t \leq 1} \int_{0}^{1}|G(t, s)| d s=\frac{1}{12}, \quad M_{1}=\max _{0 \leq t \leq 1} \int_{0}^{1}\left|G_{1}(t, s)\right| d s=\frac{1}{8}, \\
M_{2} & =\max _{0 \leq t \leq 1} \int_{0}^{1} G_{2}(t, s) d s=\frac{1}{2} .
\end{aligned}
$$

Example 4.1.1. (Example 7 in [6]) Consider the problem

$$
\begin{gathered}
u^{(3)}(t)=-e^{u(t)}, \quad 0<t<1, \\
u(0)=u^{\prime}(0)=u^{\prime}(1)=0 .
\end{gathered}
$$

Yao and Feng [6] using the lower and upper solutions method and the fixed point theorem on cones proved that the above problem has a solution $u(t)$ such that $\|u\| \leq 1, u(t)>0$ for $t \in(0,1)$ and $u(t)$ is an increasing function. Using the theoretical results obtained in the previous section, we establish the results which are stronger than the above results. Indeed, for problem (4.1) $f=f(t, x)=-e^{x}$. In

$$
\mathscr{D}_{M}^{+}=\left\{(t, x) \mid 0 \leq t \leq 1,0 \leq x \leq \frac{M}{12}\right\}
$$

there holds $-e^{M / 12} \leq f(t, x) \leq 0$. So, with the choice $M=1.1$, we have $-M \leq f(t, x) \leq 0$. Further, in $\mathscr{D}_{M}^{+}$, the function $f(t, x)$ satisfies the Lipschitz condition with $L_{0}=e^{\bar{M} / 12}=1.096$. 


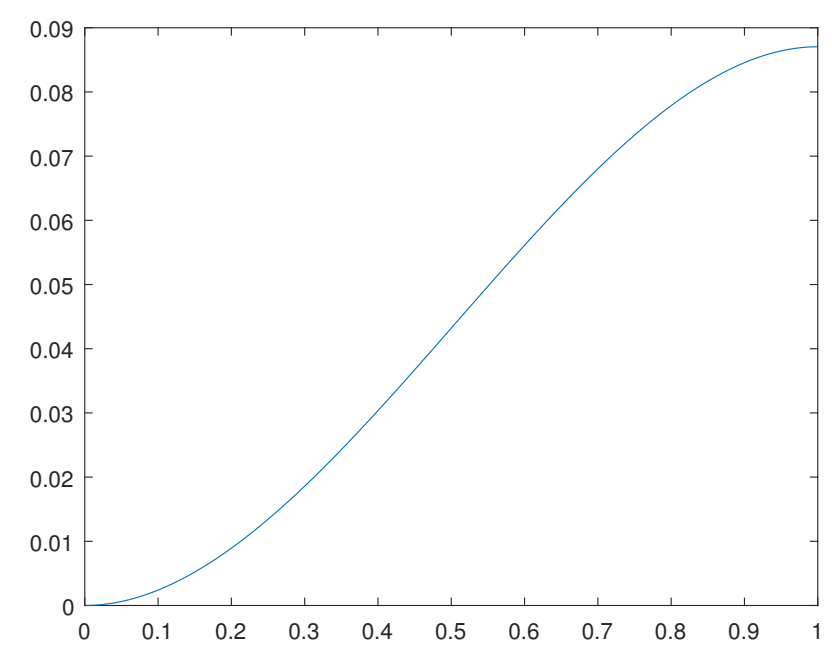

FIGURE 1. The graph of the approximate solution in Example 4.1.1.

Therefore, $q=L_{0} / 12=0.0913$. By Theorem 2.5, the problem has a unique monotone positive solution $u(t)$ satisfying the estimates

$$
\begin{aligned}
& 0 \leq u(t) \leq \frac{M}{12}=\frac{1.1}{12}=0.0917,0 \leq u^{\prime}(t) \leq \frac{M}{8}=\frac{1.1}{8}=0.1357 \\
& \left|u^{\prime \prime}(t)\right| \leq \frac{M}{2}=\frac{1.1}{2}=0.55
\end{aligned}
$$

Clearly, these results are better than those in [6].

The numerical solution of the problem obtained by the iterative method (3.1)-(3.5) after 5 iterations is depicted in Figure 1. From this figure, it is clear that the solution is monotone, positive and bounded by 0.0917 as shown above by the theory.

Example 4.1.2. (Example 8 in [6]) Consider the problem

$$
\begin{aligned}
u^{(3)}(t) & =-\frac{5 u^{3}(t)+4 u(t)+3}{u^{2}(t)+1}, \quad 0<t<1, \\
u(0) & =u^{\prime}(0)=u^{\prime}(1)=0 .
\end{aligned}
$$

Yao and Feng in [6] showed that the above problem has a solution $u(t)$ such that $u(t)>0$ for $t \in(0,1)$ and $u(t)$ is an increasing function. Similarly, as in Example 4.1.1, we establish that the problem (4.2) has a unique monotone positive solution $u(t)$ satisfying

$$
0 \leq u(t) \leq 0.3417,0 \leq u^{\prime}(t) \leq 0.5125,\left|u^{\prime \prime}(t)\right| \leq 2.05 \text {. }
$$

The numerical solution of the problem obtained by the iterative method (3.1)-(3.5) after 8 iterations is depicted in Figure 2. From this figure, it is clear that the solution is monotone, positive and bounded by 0.3417 as shown above by the theory.

Example 4.1.3. (Example 4.2 in [4]) Consider the problem

$$
\begin{aligned}
u^{(3)}(t) & =-e^{u(t)}-e^{u^{\prime}(t)}, \quad 0<t<1, \\
u(0) & =u^{\prime}(0)=u^{\prime}(1)=0 .
\end{aligned}
$$




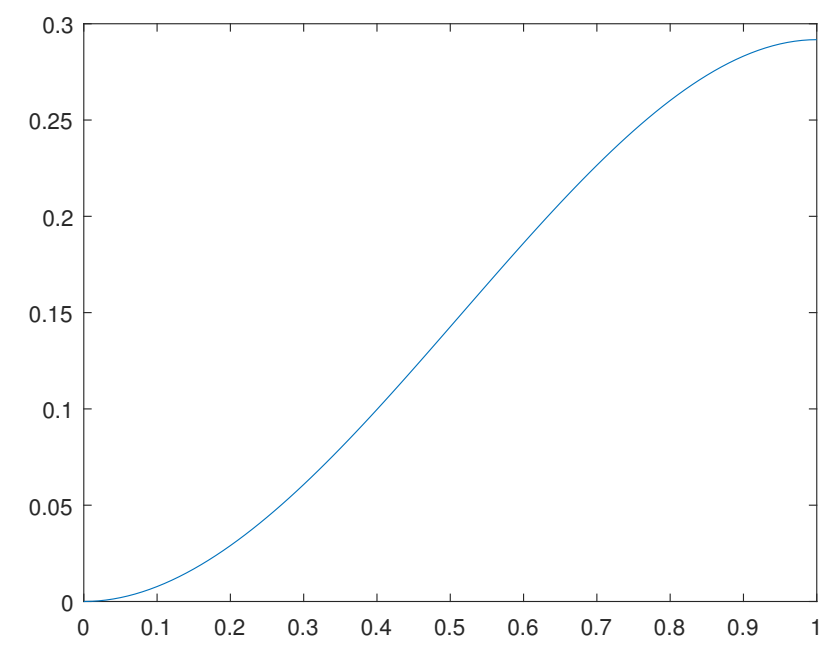

FIGURE 2. The graph of the approximate solution in Example 4.1.2.

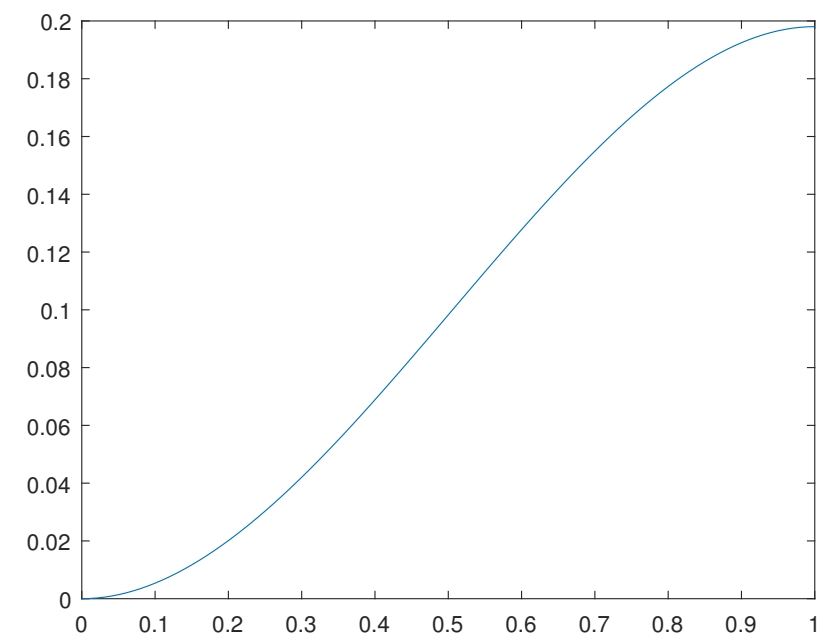

FIGURE 3. The graph of the approximate solution in Example 4.1.3.

Using the lower and upper solutions method and a new maximum principle, Feng and Liu in [4] established that the above problem has a solution $u(t)$ such that $\|u\| \leq 1, u(t)>0$ for $t \in(0,1)$ and $u(t)$ is an increasing function. Here, using Theorem 2.5 with the choice $M=2.7$, we conclude that the problem has a unique monotone positive solution $u(t)$ satisfying the estimates

$$
0 \leq u(t) \leq 0.2250,0 \leq u^{\prime}(t) \leq 0.3375,\left|u^{\prime \prime}(t)\right| \leq 1.350
$$

The numerical solution of the problem obtained by the iterative method (3.1)-(3.5) after 9 iterations is depicted in Figure 3. From this figure, it is clear that the solution is monotone, positive and bounded by 0.2250 as shown above by the theory. 
Remark 4.1. In the above examples, it is easy to see that all the conditions of Theorem 3.2 are satisfied. Therefore, the sequences of approximations are increasing. This fact is also confirmed by the numerical experiments.

Remark 4.2. It should be emphasized that in [4] and [6] the authors used one very important assumption, which means that the nonlinear functions $f(t, x)$ or $f(t, x, y)$ satisfy the one-side Lipschitz condition in $x$ or $x, y$ in the whole space $\mathbb{R}$ or $\mathbb{R}^{2}$, respectively. If we change the sign of the right-hand sides, then this condition is not satisfied. Therefore, it is impossible to say anything about the solution of the problem. But Theorem 2.4 ensures the existence and uniqueness of a solution. Moreover, in a similar way, as in Theorem 2.4, it is possible conclude that this solution is nonpositive.

4.2. Case 2. Consider the problem

$$
\begin{aligned}
u^{(3)}(t) & =f\left(t, u(t), u^{\prime}(t), u^{\prime \prime}(t)\right), \quad 0<t<1, \\
u(0) & =u^{\prime}(0)=u^{\prime \prime}(1)=0 .
\end{aligned}
$$

In [7], under the assumptions that the function $f(t, x, y, z)$ defined on $[0,1] \times \mathbb{R}^{3} \rightarrow \mathbb{R}$ is $L_{p^{-}}$ Caratheodory, and there exist functions $\alpha, \beta, \gamma, \delta \in L_{p}[0,1], p \geq 1$, such that

$$
|f(t, x, y, z) \leq \alpha(t) x+\beta(t) y+\gamma(t) z+\delta(t)|, \quad t \in(0,1)
$$

and

$$
A_{0}\|\alpha\|_{p}+A_{1}\|\beta\|_{p}+\|\gamma\|_{p}<1
$$

where $A_{0}, A_{1}$ are some constants depending on $p$, the problem has at least one solution. The tool used is the Leray-Schauder continuation principle. No examples are given for illustrating the theoretical results. Here, assuming that the function $f(t, x, y, z)$ is continuous, we establish the existence of unique solution by Theorem 2.5. For problem (4.3), the Green function is

$$
G(t, s)=\left\{\begin{array}{l}
-s t+\frac{s^{2}}{2}, \quad 0 \leq s \leq t \leq 1 \\
-\frac{t^{2}}{2}, \quad 0 \leq t \leq s \leq 1
\end{array}\right.
$$

The first and the second derivatives of this function are

$$
\begin{gathered}
G_{1}(t, s)= \begin{cases}-s, & 0 \leq s \leq t \leq 1 \\
-t, & 0 \leq t \leq s \leq 1,\end{cases} \\
G_{2}(t, s)= \begin{cases}0, & 0 \leq s \leq t \leq 1, \\
-1, & 0 \leq t \leq s \leq 1 .\end{cases}
\end{gathered}
$$

It is easy to see that

$$
G(t, s) \leq 0, G_{1}(t, s) \leq 0,0 \leq t, s \leq 1
$$

and

$$
\begin{aligned}
& M_{0}=\max _{0 \leq t \leq 1} \int_{0}^{1}|G(t, s)| d s=\frac{1}{3}, \quad M_{1}=\max _{0 \leq t \leq 1} \int_{0}^{1}\left|G_{1}(t, s)\right| d s=\frac{1}{2} \\
& M_{2}=\max _{0 \leq t \leq 1} \int_{0}^{1}\left|G_{2}(t, s)\right| d s=1 .
\end{aligned}
$$




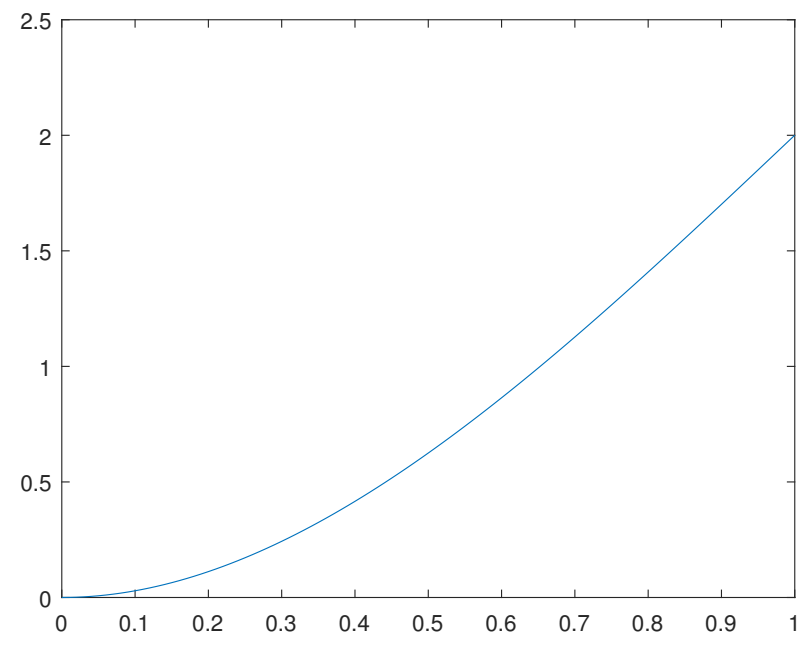

FIGURE 4. The graph of the approximate solution in Example 4.2.1.

Example 4.2.1. Consider the following problem

$$
\begin{aligned}
u^{\prime \prime \prime}(t) & =-\frac{1}{36}\left(u^{\prime}(t)\right)^{2}+\frac{1}{24} u(t) u^{\prime \prime}(t)+\frac{1}{4} t^{2}-6, \quad 0 \leq t \leq 1, \\
u(0) & =u^{\prime}(0)=u^{\prime \prime}(1)=0 .
\end{aligned}
$$

In this example

$$
f(t, x, y, z)=-\frac{1}{36} y^{2}+\frac{1}{24} x z+\frac{1}{4} t^{2}-6,
$$

it is possible to verify that with $M=7.5, L_{1}=0.3125, L_{2}=0.2083, L_{3}=0.1042$. So, all the conditions of Theorem 2.5 are satisfied, and problem (4.4) has a unique positive solution satisfying the estimates $0 \leq u(t) \leq 2.5,0 \leq u^{\prime}(t) \leq 3.75,\left|u^{\prime \prime}(t)\right| \leq 7.5$.

The numerical solution of the problem obtained by the iterative method (3.1)-(3.5) after 5 iterations is depicted in Figure 4. From this figure, it is clear that the solution is bounded by 2.5 as shown above by the theory.

It is interesting that problem (4.4) has the exact solution $u(t)=-t^{3}+3 t^{2}$.

This solution satisfies the exact estimates $0 \leq u(t) \leq 2,0 \leq u^{\prime}(t) \leq 3,0 \leq u^{\prime \prime}(t) \leq 6$ for $0 \leq t \leq 1$, which are better than the theoretical estimates above. On the grid with the gridsize $h=0.01$ the maximal deviation of the obtained approximate solution and the exact solution is $3.7665 e-04$.

4.3. Case 3. Consider the problem

$$
\begin{aligned}
u^{(3)}(t) & =f\left(t, u(t), u^{\prime}(t), u^{\prime \prime}(t)\right), \quad 0<t<1, \\
u(0) & =u^{\prime}(1)=u^{\prime \prime}(1)=0 .
\end{aligned}
$$

Under the conditions similar to those in the previous case, Hopkins and Kosmatove in [7] established the existence of a solution of the problem without illustrative examples. Recently, $\mathrm{Li}$ and $\mathrm{Li}$ [9] studied the existence of positive solutions of problem (4.5) under conditions on the growth of the function $f(t, x, y, z)$ as $|x|+|y|+|z|$ tends to zero and infinity, including a Nagumo-type condition on $y$ and $z$. The tool used is the fixed point index theory on cones. 
Here, assuming that the function $f(t, x, y, z)$ is continuous, we can establish the existence results by the theorems in the Section 2. For problem (4.5), the Green function is

$$
G(t, s)=\left\{\begin{array}{l}
\frac{s^{2}}{2}, \quad 0 \leq s \leq t \leq 1, \\
s t-\frac{t^{2}}{2}, \quad 0 \leq t \leq s \leq 1 .
\end{array}\right.
$$

The first and the second derivatives of this function are

It is easy to see that

$$
\begin{aligned}
& G_{1}(t, s)= \begin{cases}0, & 0 \leq s \leq t \leq 1, \\
s-t, & 0 \leq t \leq s \leq 1,\end{cases} \\
& G_{2}(t, s)= \begin{cases}0, & 0 \leq s \leq t \leq 1, \\
-1, & 0 \leq t \leq s \leq 1 .\end{cases}
\end{aligned}
$$

$$
G(t, s) \geq 0, G_{1}(t, s) \geq 0,0 \leq t, s \leq 1
$$

and

$$
M_{0}=\frac{1}{6}, M_{1}=\frac{1}{2}, M_{2}=1 .
$$

Example 4.3.1. Consider the following problem

$$
\begin{aligned}
u^{\prime \prime \prime}(t) & =\frac{1}{18}\left(u^{\prime}(t)\right)^{2}-\frac{1}{12} u(t) u^{\prime \prime}(t)+\frac{1}{2} t+\frac{11}{2}, \quad 0 \leq t \leq 1, \\
u(0) & =u^{\prime}(1)=u^{\prime \prime}(1)=0 .
\end{aligned}
$$

In this example,

$$
f(t, x, y, z)=\frac{1}{18} y^{2}-\frac{1}{12} x z+\frac{1}{2} t+\frac{11}{2} .
$$

It is possible to verify that with $M=8, L_{1}=\frac{2}{3}, L_{2}=\frac{4}{9}, L_{3}=\frac{1}{9}$, and all the conditions of Theorem 2.5 are satisfied. Therefore, problem (4.6) has a unique positive solution, which is increasing and satisfies the estimates $0 \leq u(t) \leq \frac{4}{3}, 0 \leq u^{\prime}(t) \leq 4,-8 \leq u^{\prime \prime}(t) \leq 0$. The numerical solution of the problem obtained by the iterative method (3.1)-(3.5) after 6 iterations is depicted in Figure 5. From this figure, it is clear that the solution is monotone, positive and bounded by $4 / 3$ as shown above by the theory.

It is possible to verify that the function $u(t)=t^{3}-3 t^{2}+3 t$ is the exact solution of problem (4.6). This solution is positive, increasing and satisfies the exact estimates $0 \leq u(t) \leq 1,0 \leq$ $u^{\prime}(t) \leq 3,-6 \leq u^{\prime \prime}(t) \leq 0$ for $0 \leq t \leq 1$, which are better than the theoretical estimates above. On the grid with the gridsize $h=0.01$ the maximal deviation of the obtained approximate solution and the exact solution is $3.6256 e-04$.

4.4. Case 4. Consider the problem

$$
\begin{aligned}
u^{(3)}(t) & =f\left(t, u(t), u^{\prime}(t), u^{\prime \prime}(t)\right), \quad 0<t<1, \\
u(0) & =u^{\prime \prime}(0)=u^{\prime}(1)=0 .
\end{aligned}
$$

Using the lower and upper solutions method and Schauder fixed theorem on cones, Bai [10] established the existence of a solution under complicated conditions on the right-hand side function. 


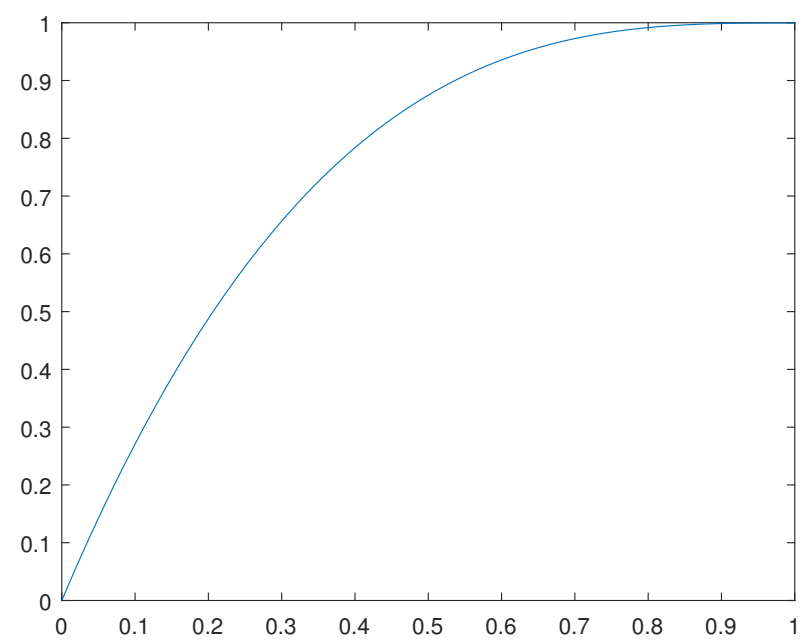

FIGURE 5. The graph of the approximate solution in Example 4.3.1.

For problem (4.7), the Green function is

$$
G(t, s)=\left\{\begin{array}{l}
\frac{t^{2}}{2}-t+\frac{s^{2}}{2}, \quad 0 \leq s \leq t \leq 1 \\
t(s-1), \quad 0 \leq t \leq s \leq 1
\end{array}\right.
$$

The first and the second derivatives of this function are

$$
\begin{gathered}
G_{1}(t, s)= \begin{cases}t-1, & 0 \leq s \leq t \leq 1 \\
s-1, & 0 \leq t \leq s \leq 1\end{cases} \\
G_{2}(t, s)= \begin{cases}1, & 0 \leq s \leq t \leq 1 \\
0, & 0 \leq t \leq s \leq 1\end{cases}
\end{gathered}
$$

Obviously,

$$
G(t, s) \leq 0, G_{1}(t, s) \leq 0,0 \leq t, s \leq 1
$$

and it is easy to obtain

$$
M_{0}=\frac{1}{3}, M_{1}=\frac{1}{2}, M_{2}=1 .
$$

In view of the above facts concerning the Green function, using theorems in the previous section, we can establish the results on the existence of solution of the problem (4.7).

Example 4.4.1 (Example 3.5 in [10])

$$
\begin{aligned}
u^{(3)}(t) & =-\frac{1}{4}\left[t+e^{u(t)}+\left(u^{\prime}(t)\right)^{2}+u^{\prime \prime}(t)\right], \quad 0<t<1, \\
u(0) & =u^{\prime \prime}(0)=u^{\prime}(1)=0 .
\end{aligned}
$$

Defining

$$
\mathscr{D}_{M}^{+}=\left\{(t, x, y, z)\left|0 \leq t \leq 1,0 \leq x \leq \frac{M}{3}, 0 \leq y \leq \frac{M}{2},\right| z \mid \leq M\right\}
$$




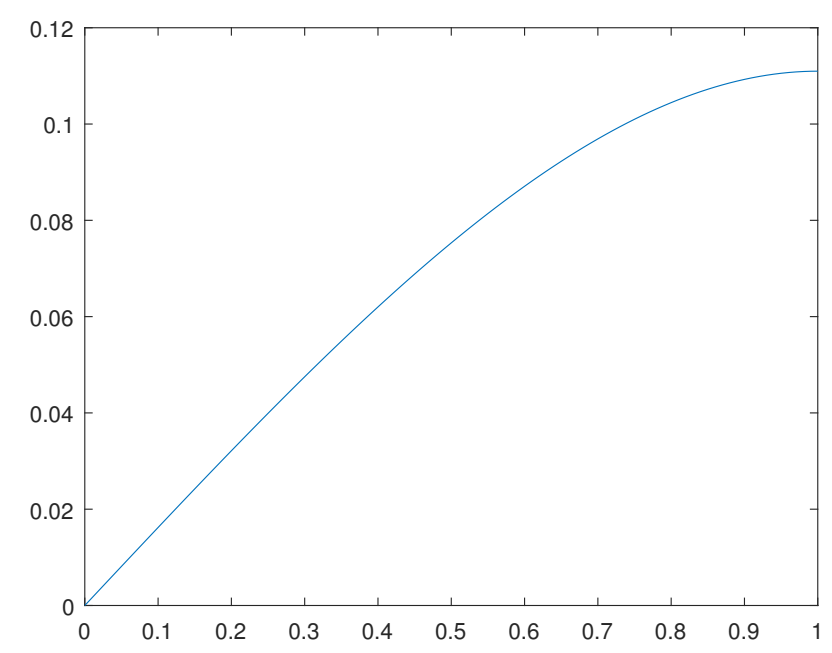

FIGURE 6. The graph of the approximate solution in Example 4.4.1.

for $M=0.835$, we have

$$
-M \leq f(t, x, y, z)=-\frac{1}{4}\left[t+e^{x}+y^{2}+z\right] \leq 0
$$

Further, it is easy to calculate the Lipschitz coefficients of $f(t, x, y, z)$ :

$$
L_{0}=\frac{1}{4} e^{M / 3}=0.3302, L_{1}=\frac{M}{4}=0.2087, L_{2}=1 .
$$

Therefore, $q=L_{0} / 3+L_{1} / 2+L_{2}=0.4851<1$. By Theorem 2.5, the problem has a unique monotone positive solution $u(t)$ such that

$$
0 \leq u(t) \leq M / 3=0.2783,0 \leq u^{\prime}(t) \leq M / 2=0.5,\left|u^{\prime \prime}(t)\right| \leq 1 .
$$

Notice that in [10] Bai could only conclude that problem (4.8) has a positive solution. The numerical solution of the problem obtained by the iterative method (3.1)-(3.5) after 5 iterations is depicted in Figure 6. From this figure, it is clear that the solution is monotone, positive and bounded by 0.2783 as shown above by the theory.

4.5. Case 5. Consider the problem

$$
\begin{aligned}
u^{(3)}(t) & =f\left(t, u(t), u^{\prime}(t), u^{\prime \prime}(t)\right), \quad 0<t<1, \\
u(0) & =u^{\prime}(1)=u^{\prime \prime}(1)=0,
\end{aligned}
$$

In [9], based on the fixed point index theory in cones, they established the existence of positive solution under complicated conditions posed on the growth of the function $f$ including a Nagumo-type condition. For problem (4.9), the Green function is

$$
G(t, s)=\left\{\begin{array}{l}
\frac{s^{2}}{2}, \quad 0 \leq s \leq t \leq 1 \\
s t-\frac{t^{2}}{2}, \quad 0 \leq t \leq s \leq 1
\end{array}\right.
$$


The first and the second derivatives of this function are

$$
\begin{gathered}
G_{1}(t, s)= \begin{cases}0, & 0 \leq s \leq t \leq 1, \\
s-t, & 0 \leq t \leq s \leq 1,\end{cases} \\
G_{2}(t, s)= \begin{cases}0, & 0 \leq s \leq t \leq 1, \\
-1, & 0 \leq t \leq s \leq 1 .\end{cases}
\end{gathered}
$$

It is easy to see that

$$
G(t, s) \geq 0, G_{1}(t, s) \geq 0, G_{2}(t, s) \leq 0,0 \leq t, s \leq 1
$$

and

$$
M_{0}=\frac{1}{6}, M_{1}=\frac{1}{2}, M_{2}=1 .
$$

In view of the above facts concerning the Green function, using theorems in the previous section, we can establish the results on the existence of solution of the problem (4.9).

Example 4.5.1. Consider the following problem

$$
\begin{aligned}
u^{\prime \prime \prime}(t) & =\frac{1}{18}\left(u^{\prime}(t)\right)^{2}-\frac{1}{12} u(t) u^{\prime \prime}(t)+\frac{1}{2} t+\frac{11}{2}, \quad 0 \leq t \leq 1 \\
u(0) & =u^{\prime}(1)=u^{\prime \prime}(1)=0
\end{aligned}
$$

In this example,

$$
\begin{aligned}
& f(t, x, y, z)=\frac{1}{18} y^{2}-\frac{1}{12} x z+\frac{1}{2} t+\frac{11}{2} \\
& f(t, 0,0,0)=\frac{1}{2} t+\frac{11}{2}>0 \quad \forall t \in[0,1] .
\end{aligned}
$$

It is easy to verify that with $M=8$ all the conditions of Theorem 2.4 are satisfied due to the fact that this the problem has a unique positive increasing solution satisfying the estimates $0 \leq u(t) \leq \frac{4}{3}, 0 \leq u^{\prime}(t) \leq 4,\left|u^{\prime \prime}(t)\right| \leq 8$. Notice that the function $u(t)=t^{3}-3 t^{2}+3 t$ is the exact solution of problem (4.10). This solution is positive, increasing and satisfies the exact estimates $0 \leq u(t) \leq 1,0 \leq u^{\prime}(t) \leq 3,-6 \leq u^{\prime \prime}(t) \leq 0$ for $0 \leq t \leq 1$, which are better than the theoretical estimates above.

Example 4.5.2. Consider the following problem

$$
\begin{aligned}
u^{\prime \prime \prime}(t) & =u^{3}(t)+u(t)\left(u^{\prime}(t)\right)^{2}+u(t)\left(u^{\prime \prime}(t)\right)^{2}, 0 \leq t \leq 1 \\
u(0) & =u^{\prime}(1)=u^{\prime \prime}(1)=0
\end{aligned}
$$

In this example,

$$
f(t, x, y, z)=x^{3}+x y^{2}+x z^{2}
$$

It is easy to verify that with $0<M \leq \sqrt{\frac{108}{23}}$ Theorem 2.5 guarantees that problem (4.11) has a unique nonnegative monotone solution. Because $u(t) \equiv 0$ is a nonnegative solution of the problem, we conclude that the problem cannot have positive solution. This conclusion is in contrast with the conclusion in [9]. Therefore, there may be an inaccuracy in their results. 


\section{CONCLUSION}

In this paper, we proposed a novel efficient approach to study fully third order differential equations with general two-point linear boundary conditions. The approach is based on the reduction of boundary value problems to fixed point problems for nonlinear operators on the right-hand sides of the equation but not for the function to be sought. The results are that we established the existence, uniqueness, positivity and monotonicity of solutions under the conditions, which are simpler and easier to verify. The applicability and advantages of the proposed approach are illustrated by some known examples, where our approach gives better results. Our proposed approach can be applicable to other boundary value problems for the third order and higher orders nonlinear differential equations. This is the subject of our research in the future.

\section{APPENDIX}

In the space $C[a, b]$, we consider the operator $y=A x$ defined by the formula

$$
y(t)=\int_{a}^{b} K(t, s) x(s) d s .
$$

Theorem 5.1. [21, Secion 31] The above formula defines a compact operator in the space $C[a, b]$ if the function $K(t, s)$ is bounded on the square $a \leq t \leq b, a \leq s \leq b$ and all points of discontinuity of the function $K(s, t)$ lie on a finite number of curves

$$
s=\varphi_{k}(t), \quad k=1,2 \ldots, n,
$$

where $\varphi_{k}(t)$ the are continuous functions.

\section{Acknowledgements}

The first author was supported by Vietnam National Foundation for Science and Technology Development (NAFOSTED) under the grant number 102.01-2017.306. The second author was supported by Institute of Information Technology, VAST under the project CS 20.01.

\section{REFERENCES}

[1] A. Cabada, The method of lower and upper solutions for second, third, forth, and higher order boundary value problems, J. Math. Anal. Appl. 185 (1994), 302-320.

[2] A. Cabada, The method of lower and upper solutions for third-order periodic boundary value problems, J. Math. Anal. Appl. 195 (1995), 568-589.

[3] Y. Feng, Solution and positive solution of a semilinear third-order equation, J. Comput. Appl. Math. 29 (2009), 153-161.

[4] Y. Feng, S, Liu, Solvability of a third-order two-point boundary value problem, Appl. Math. Lett. 18 (2005), 1034-1040.

[5] M.R. Grossinho, F. Minhos, Existence result for some third order separated boundary value problems, Nonlinear Anal. 47 (2001), 2407-2418.

[6] Q. Yao, Y. Feng, The existence of solutions for a third order two-point boundary value problem, Appl. Math. Lett. 15 (2002), 227-232.

[7] B. Hopkins, N. Kosmatov, Third-order boundary value problems with sign changing solutions, Nonlinear Anal. 67 (2007), 126-137.

[8] Y. Li, Positive periodic solutions for fully third-order ordinary differential equations, Comput. Math. Appl. 59 (2010), 3464-3471. 
[9] Y. Li, Y. Li, Positive solutions of a third-order boundary value problem with full nonlinearity, Mediterr. J. Math. 14 (2017), 128.

[10] Z. Bai, Existence of solutions for some third-order boundary-value problems, Electron. J. Differential Equations 2008 (2008), 25

[11] Y. Sun, M. Zhao, S. Li, Monotone positive solution of nonlinear third-order two-point boundary value problem, Miskolc Math. Notes 15 (2014), 743-752.

[12] Q.A. Dang, T.K.Q. Ngo, Existence results and iterative method for solving the cantilever beam equation with fully nonlinear term, Nonlinear Anal. Real World Appl. 36 (2017), 56-68.

[13] Q.A. Dang, Q.L. Dang, T.K.Q. Ngo, A novel efficient method for nonlinear boundary value problems, Numer. Algo. 76 (2017), 427-439.

[14] Q.A. Dang, T.H. Nguyen, The unique solvability and approximation of BVP for a nonlinear fourth order Kirchhoff type equation, East Asian J. Appl. Math. 8 (2018), 323-335.

[15] Q.A. Dang, K.Q. Ngo, New fixed point approach for a fully nonlinear fourth order boundary value problem, Bol. Soc. Paran. Mat. 36 (2018), 209-223.

[16] Q.A. Dang, T.H. Nguyen, Existence results and numerical method for a fourth order nonlinear problem, Int. J. Appl. Comput. Math. 4 (2018), 148.

[17] Q.A. Dang, Q.L. Dang, A simple efficient method for solving sixth-order nonlinear boundary value problems, Comp. Appl. Math. 37 (2018), 16-26.

[18] Q.A. Dang, T.H. Nguyen, Existence results and iterative method for solving a nonlinear biharmonic equation of Kirchhoff type, Comput. Math. Appl. 76 (2018), 11-22.

[19] Z. Bai, Z. Du, S. Zhang, Iterative method for a class of fourth-order P-Laplacian beam equation, J. Appl. Anal. Comput. 9 (2019), 1443-1453.

[20] Y. Wei Q. Song, Z. Bai, Existence and iterative method for some fourth order nonlinear boundary value problems, Appl. Math. Lett. 87 (2019), 101-107.

[21] N. Kolmogorov, S.V. Fomin, Elements of the Theory of Functions and Functional Analysis, Vol.1, Metric and Normed Spaces, Graylock Press, Rochester, NY, 1957.

[22] E. Zeidler, Nonlinear Functional Analysis: I: Fixed-Point Theorems, Springer, Berlin, 1986. 